\title{
Adenovirus type 5 induces vitamin A-metabolizing enzymes in dendritic cells and enhances priming of gut-homing CD8 T cells
}

\author{
S Ganguly ${ }^{1}$, S Manicassamy ${ }^{3}$ J Blackwell ${ }^{2,4}$, B Pulendran ${ }^{3}$ and RR Amara ${ }^{1,4}$
}

\begin{abstract}
Protective immunity at the gut-associated mucosal tissue is induced primarily by oral/rectal immunization owing to the need for targeting antigen to the gut-resident dendritic cells (DCs). In this study we show that an adenovirus type 5 (Ad5)-based human immunodeficiency virus type 1 vaccine can prime a durable antigen-specific CD8 T-cell response in the gut following intramuscular (IM) immunization in mice. The ability of Ad5 to prime gut-homing CD8 T cells in vivo was associated with Ad5-induced expression of retinal dehydrogenase (RALDH) enzymes in conventional DCs. The Ad5-mediated induction of RALDH did not require signaling through Toll-like receptors, DNA-dependent activator of interferon regulatory factors and several mitogen-activated protein kinases, or replication capacity of the virus, but was dependent on nuclear factor- $\kappa B$ and granulocyte-macrophage colony-stimulating factor. These results provide an innate mechanism through which Ad5-stimulated DCs prime gut-homing CD8 T cells and have implications for the development of novel mucosal adjuvants for subunit vaccines administered via the IM route.
\end{abstract}

\section{INTRODUCTION}

Pathogenic human immunodeficiency virus (HIV)/Simian immunodeficiency virus infections are characterized by the rapid depletion of CD4 $\mathrm{T}$ cells in the gastrointestinal tract within 2 weeks following infection, and vaccination strategies that induce high levels of antiviral immunity at the gut-associated mucosal tissue can significantly enhance protection. Oral/rectal routes of vaccination is the best way to induce immunity at the gut-associated mucosal tissue. ${ }^{1,2}$ However, both routes of vaccinations are limited by requiring multiple high doses of the vaccine and the need to use adjuvants for induction of an optimal immune response.

The need for oral/rectal immunizations to elicit protective immunity at gut-associated mucosal tissue was attributed to the findings that gut-resident dendritic cells (DCs) have an intrinsic capacity of metabolizing vitamin A to retinoic acid (RA) that is required for imprinting gut-homing potential on $\mathrm{T}$ and B lymphocytes. ${ }^{3,4}$ Synthesis of RA depends on the oxidative metabolism of retinol to retinal that requires alcohol dehydrogenases and then conversion of retinal to RA that requires retinal dehydrogenases (RALDHs). The gut-resident DCs possess the property to synthesize RA because they constitutively express RALDH enzymes. ${ }^{3}$ It was previously thought that peripheral DCs do not constitutively express RALDH enzymes and thus are incapable of imprinting gut-homing phenotype on $\mathrm{T}$ and $\mathrm{B}$ cells. A recent study has, however, shown that under steady-state conditions, RA-producing DCs can also be found in the skin, lungs, and the corresponding draining lymph nodes (DLNs) of these tissues. ${ }^{5}$

In recent past, some studies have demonstrated that intramuscular (IM) immunizations with live replication-defective/ attenuated recombinant viral vectors such as adenovirus type 5 (Ad5) and MVA (modified vaccinia Ankara) can elicit immune responses in the gut-associated mucosal tissue in the murine ${ }^{6,7}$ and macaque ${ }^{8}$ models, suggesting that a potent gut mucosal immunity is achievable with a parenteral route of immunization. Similarly, acute LCMV (lymphocytic choriomeningitis virus) infection of mice has been shown to induce antiviral CD8 T cells capable of trafficking to gut within days after infection. ${ }^{9}$ However, the mechanisms by which parenteral immunizations

\footnotetext{
${ }^{1}$ Department of Microbiology and Immunology, Emory Vaccine Center, Emory University School of Medicine, Atlanta, Georgia, USA. ${ }^{2}$ Division of Infectious Disease, Emory Vaccine Center, Emory University School of Medicine, Atlanta, Georgia, USA. ${ }^{3}$ Department of Pathology, Emory Vaccine Center, Emory University School of Medicine, Atlanta, Georgia, USA. ${ }^{4}$ Division of Microbiology and Immunology, Yerkes National Primate Research Center, Emory University, Atlanta, Georgia, USA.

Correspondence: RR Amara (ramara@emory.edu)

Received 12 April 2010; accepted 1 January 2011; published online 2 February 2011. doi:10.1038/mi.2011.1
} 
induce antigen-specific CD8 T cells with gut-homing potential are not understood. It is possible that these viruses modulate the function of peripheral DCs such that they acquire the capacity to induce gut-homing potential on antigen-specific CD8 T cells. Furthermore, recently the STEP trial evaluating the efficacy of an Ad5-based HIV type 1 (HIV-1) vaccine was halted because of the presumed increased risk of HIV-1 acquisition in men who were baseline Ad5 seropositive and uncircumcised. ${ }^{10}$ It was hypothesized that this increased risk could be because of increased frequency of virus target cells at the mucosa primed by Ad5. Thus, defining the mechanisms by which Ad5 induces gut-homing potential on $\mathrm{T}$ cells is important for the understanding of viral vector-based HIV vaccines.

In this study we investigated the mechanisms by which Ad5 can modulate the function of peripheral DCs to induce guthoming potential on CD8 T cells. Our results demonstrate that Ad5 rapidly upregulates the expression of RALDH enzymes in conventional DCs (cDCs) that results in priming of antigenspecific CD8 T cells that coexpress gut-homing marker $\alpha_{4} \beta_{7}$. Impressively, this function of Ad5 is independent of signaling through Toll-like receptors (TLRs), DNA-dependent activator of interferon regulatory factors (DAI; previously also known as ZBP-1 (Z-DNA binding protein 1)), and some mitogen-activated protein (MAP) kinases, but was dependent on granulocytemacrophage colony-stimulating factor (GM-CSF) and nuclear factor- $\kappa \mathrm{B}(\mathrm{NF}-\kappa \mathrm{B})$ in DCs.

\section{RESULTS}

Parenteral immunization with Ad5/Env-Gag vaccine can induce gut-homing potential on antigen-specific CD8 T cells

To determine whether IM administered recombinant adenovirus serotype 5 vaccine vector can induce gut-homing potential on antigen-specific CD8 T cells, BALB/c mice were primed and boosted with $1 \times 10^{6} \mathrm{PFU}$ of the Ad5 expressing HIV-1 clade B Env and Gag (Ad5/Env-Gag) at weeks 0 and 4, respectively. The Gag-specific CD8 T-cell responses in peripheral blood and lamina propria lymphocyte (LPL) population in the small intestines (will be referred to as "Gut" hereafter) were evaluated using a $\mathrm{H}$ $2 \mathrm{~K}^{\mathrm{d}}$-restricted Gag tetramer at various time points following the boost (Figure 1a and $\mathbf{b}$ ). Expansion of gut-resident Gag-specific CD8 T cells was detected as early as day 4 following the boost, peaked at day 7, and contracted and persisted as late as day 150. At the peak response, the frequency (arithmetic mean) of these cells was about $15 \%$ of total CD8 T cells and was also 15 -fold higher compared with preboost levels. Similar expansion/ contraction kinetics were also observed in blood. However, on day 4 following boost, the mean percentage of tetramer-specific CD8 T cells in peripheral blood was 3.7-fold lower compared with the gut, suggesting a preferential early homing of antigenspecific CD8 T cells to the gut upon IM immunization with Ad5/Env-Gag. By 2 weeks following the boost, the frequencies of Gag-specific CD8 T cells were similar in both compartments. These Gag-specific LPLs were also functional as evident from interferon- $\gamma$ production upon ex vivo stimulation with a Gag peptide pool that encompasses the entire Gag protein, although at a lower level compared with tetramer + cells (Figure 1c).
Interestingly, this low interferon- $\gamma$ response with respect to tetramer frequency was only true for the LPLs and not for other tissues (spleen and liver) analyzed on day 7 post boost (data not shown) as shown previously. ${ }^{8} \mathrm{Gag}$-specific CD8 T cells were also present in the intraepithelial lymphocyte population of the gut as early as day 7 following the boost (data not shown). However, the frequency of these cells was $\sim 14$-fold lower compared with their frequency in the lamina propria.

We next analyzed the expression of the integrin $\alpha_{4} \beta_{7}$ on Gag tetramer-specific cells in blood to determine whether the early gut-homing potential of these cells can be predicted from the cell surface expression of this gut-homing marker (Figure 1d and $\mathbf{e}$ ). The $\alpha_{4} \beta_{7}$ ligand, MAdCAM- 1 (mucosal addressin cell adhesion molecule-1), is expressed by the high endothelial venules in the lamina propria and is known to mediate T-cell recruitment to the gut. ${ }^{11}$ Approximately $30-40 \%$ of Gag tetramer-specific CD8 T cells in blood expressed $\alpha_{4} \beta_{7}$ as early as day 4 following the boost. However, the $\alpha_{4} \beta_{7}$ expression on these cells decreased rapidly by day 7 , and only a small fraction $(<10 \%)$ of tetramerpositive cells retained expression on day 28. A similar pattern was also observed for the per-cell expression (as determined by the mean fluorescence intensity) of $\alpha_{4} \beta_{7}$ on the tetramerpositive cells, which was highest on day 4 following the boost (Figure 1e, right panel). This high level of $\alpha_{4} \beta_{7}$ expression on tetramer-positive cells in blood on day 4 following the boost is consistent with the preferential homing of Gag-specific T cells to the gut, and thus may be used as a marker to predict the guthoming potential of CD8 T cells following parenteral immunization. We also observed an increase in Gag tetramer-specific CD8 T cells coexpressing CCR9 (chemokine (C-C motif) receptor 9; homing marker for small intestine) on day 4 following the boost (Figure 1f and $\mathbf{g}$ ). However, the percentage of cells coexpressing $\alpha_{4} \beta_{7}$ was twofold higher in comparison with those coexpressing CCR9. Our results thus suggest that both $\alpha_{4} \beta_{7}$ and CCR9 have a role in Ad5-mediated induction of gut trafficking CD8 T cells.

\section{Ad5 endows splenic $c D C s$ with the capacity to imprint gut-homing potential on antigen-specific CD8 T cells via a RA-dependent pathway}

Upon IM immunization, Ad5 can either modulate peripheral DC function or target gut-resident DCs such that they can prime antigen-specific CD8 T cells with gut-homing potential. However, it is less likely that the latter is true for this route of antigen delivery. Hence, we investigated whether the Ad5 can modulate peripheral DC function with regard to priming antigen-specific CD8 T cells with gut-homing potential using an in vitro assay system. We co-cultured the ovalbumin-specific OT-I transgenic T cells with either uninfected or Ad5 (nonrecombinant)-infected splenic cDCs in the presence of ovalbumin peptide (SIINFEKL) for $72 \mathrm{~h}$ and analyzed the expression of $\alpha_{4} \beta_{7}$ on these T cells. To avoid any extraneous virus-induced DC death during the co-culture, Ad5 infection of cDCs was carried out for $8 \mathrm{~h}$ and cells were then washed to remove cell-free virus before co-culturing with the OT-I T cells. The percentage of $\alpha_{4} \beta_{7^{-}}$ expressing OT-I CD8 T cells was 2.8-fold higher when primed with Ad5-infected cDCs (multiplicity of infection (MOI) 0.15) 
a

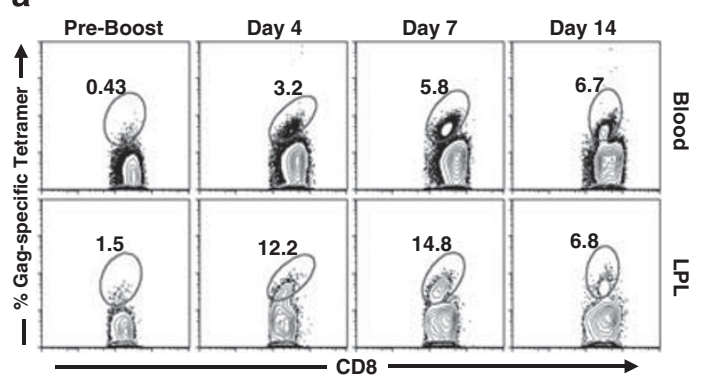

d

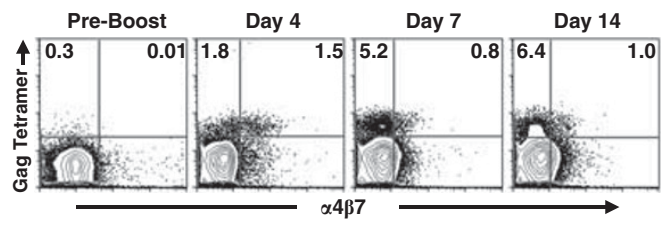

f

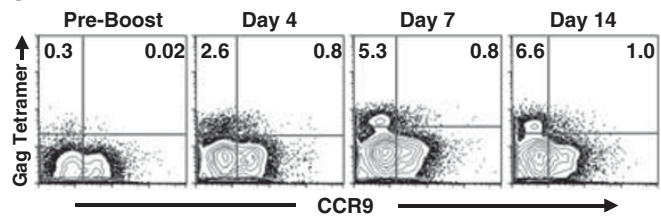

b

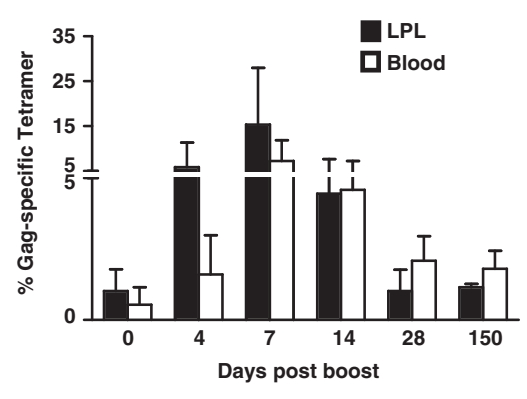

C

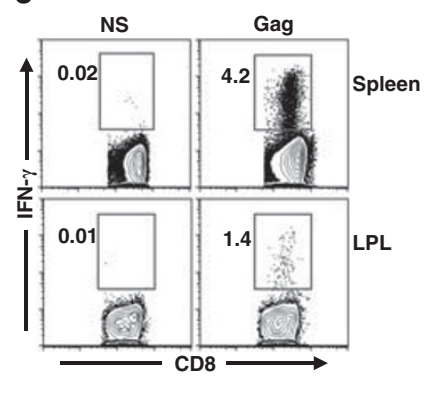

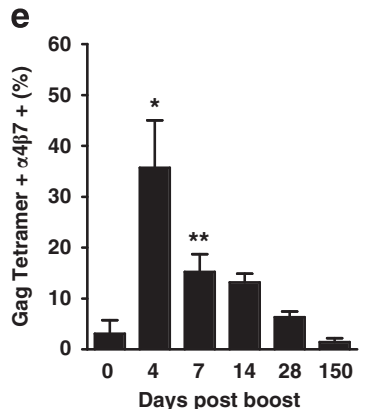

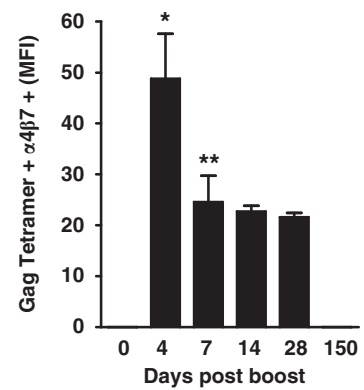

g

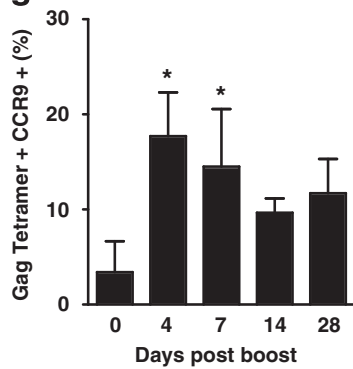

Figure 1 Parenteral immunization with Ad5/Env-Gag vaccine can induce gut-homing potential on antigen-specific CD8 T cells. BALB/c mice were primed and boosted with Ad5/Env-Gag vector at weeks 0 and 4, respectively. (a) Representative fluorescence-activated cell sorting (FACS) plot showing the frequency of Gag tetramer-specific CD8 T cells in blood and in lamina propria lymphocytes (LPLs) at different time points following the boost. The numbers on the FACS plots indicate Gag tetramer-specific CD8 T cells expressed as a percentage of total CD8 T cells. (b) Kinetics of Gag tetramer-specific CD8 T-cell response in blood and LPLs following Ad5/Env-Gag boost. (c) Representative FACS plots showing Gag-specific CD8 T-cell responses in spleen and LPLs at 1 week following the Ad5/Env-Gag boost as assessed by intracellular cytokine staining. The numbers on the FACS plots indicate Gag-specific interferon (IFN)- $\gamma^{+}$CD8 T cells expressed as a percentage of total CD8 T cells. NS, no stimulation. (d) Representative FACS plot showing $\alpha_{4} \beta_{7}$ expression on Gag tetramer-specific CD8 T cells in blood (gated on total CD8 lymphocytes). (e) Mean frequency (left panel) and mean fluorescence intensity (MFI; right panel) of $\alpha_{4} \beta_{7}{ }^{+}$Gag-tetramer ${ }^{+}$CD8 T cells in blood. (f) Representative FACS plot showing CCR9 expression on Gag tetramer-specific CD8 T cells in blood (gated on total CD8 lymphocytes). (g) Mean frequency of CCR9 ${ }^{+}$Gag tetramer ${ }^{+}$CD8 T cells in blood. Three to five mice were analyzed at each time point. Error bars indicate s.d. Data are representative of one of two independent experiments. ${ }^{\star} P<0.05 ;{ }^{\star \star} P<0.01$. The $P$-values indicate significantly higher responses compared with day 0 .

in comparison with the $\mathrm{T}$ cells primed with uninfected cDCs (Figure 2a, middle panel), demonstrating that Ad5 vector alone can enhance the ability of cDCs to imprint gut-homing potential on antigen-specific CD8 T cells. However, at higher MOI of Ad5 infection, the percentage of CD8 T cells coexpressing $\alpha_{4} \beta_{7}$ was diminished (Figure 2a, middle panel). Importantly, splenic $\mathrm{cDCs}$ infected with higher MOIs failed to prime CD8 $\mathrm{T}$ cells as efficiently as those infected with a lower MOI (0.15) or uninfected cDCs as evident from the CD8 T-cell viability data after $72 \mathrm{~h}$ of co-culture (Figure 2a, right panel), suggesting that higher MOI of Ad5 may be reducing cDC viability. The Ad5mediated effect on induction of $\alpha_{4} \beta_{7}$ was also true for CCR9, as following in vitro infection of splenic cDCs (MOI 2), the percentage of CD8 T cells coexpressing CCR9 was 2.5 -fold higher in comparison with $\mathrm{CD} 8 \mathrm{~T}$ cells that were primed with uninfected cDCs (Figure 2b). As expected, co-culture of T cells with RA induced highest levels of $\alpha_{4} \beta_{7}$ and CCR9 on OT-I T cells.

Previous studies ${ }^{3,4}$ have shown that GALT (gut-associated lymphoid tissue) DCs have the intrinsic capacity to metabolize vitamin A (retinol) to RA that in turn imprints gut-homing potential on naive/responding $\mathrm{T}$ and $\mathrm{B}$ cells. Hence, we investigated whether induction of gut-homing potential on antigenspecific CD8 T cells by Ad5-infected cDCs was a RA-dependent or an independent effect. Intracellularly, RA signals through either the heterodimeric (RAR/RXR) or homodimeric (RXR/ RXR) RA receptors (RARs) and functions as ligand-induced transcription factor by interacting with RA response elements (RAREs) or RX response elements (RXREs) located in the 
a

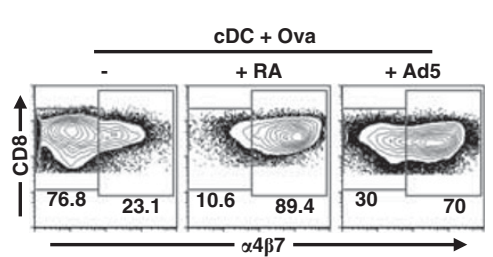

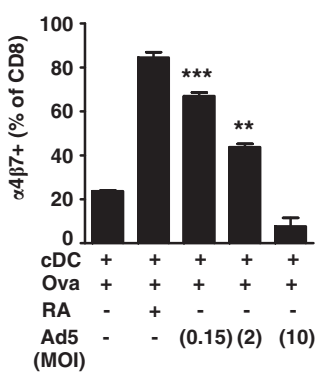

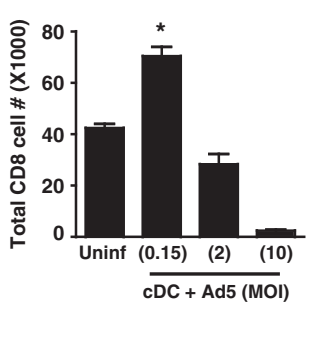

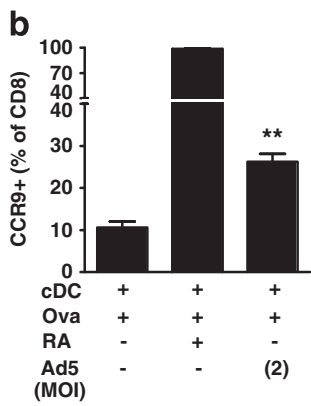
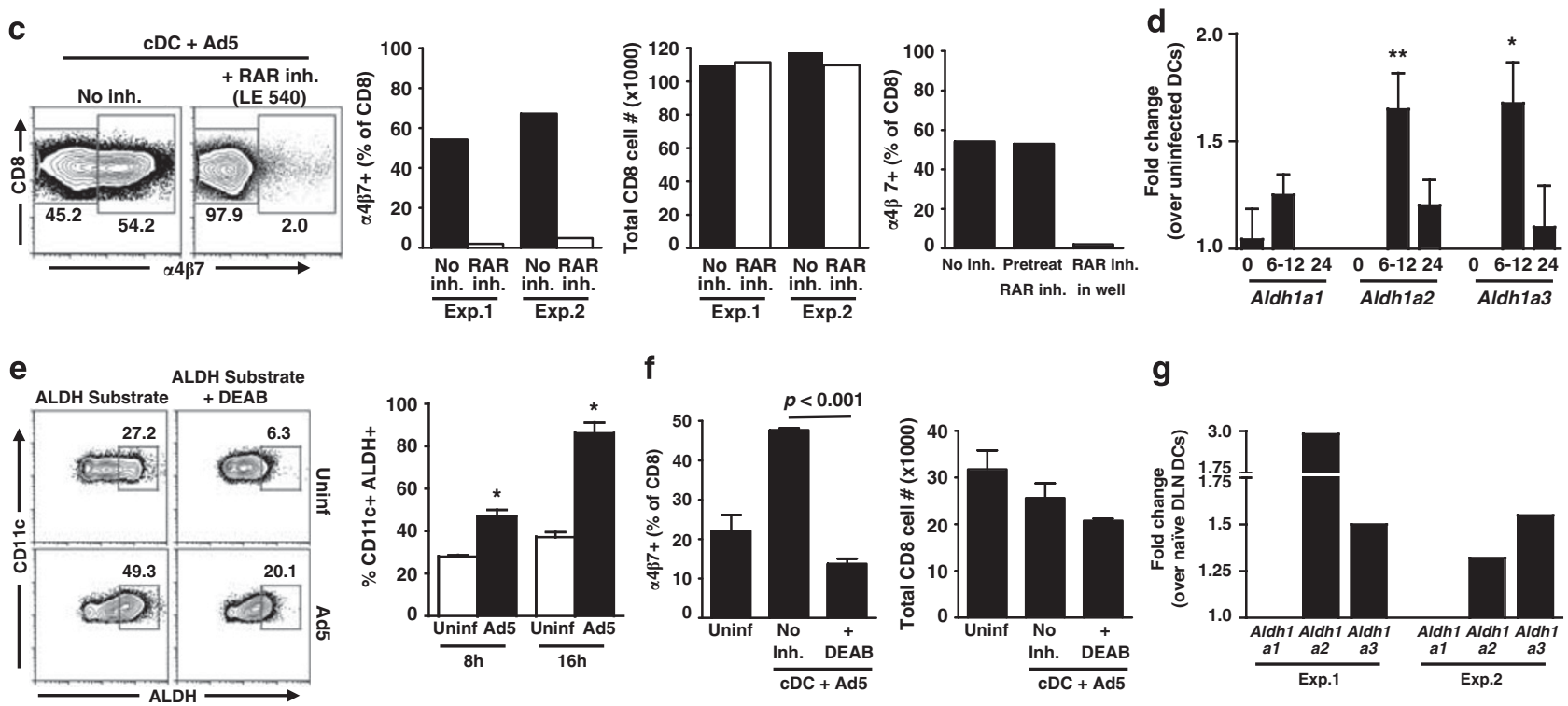

$\mathbf{f}$

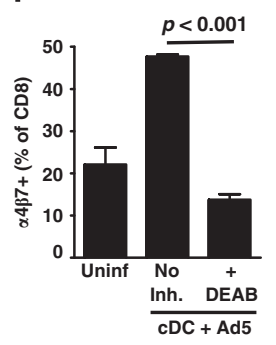

g

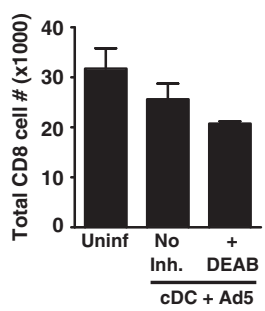

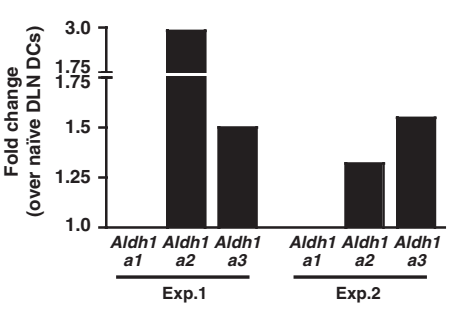

Figure 2 Adenovirus type 5 (Ad5) endows splenic conventional dendritic cells (cDCs) with the capacity to imprint gut-homing potential on antigenspecific CD8 T cells via a retinoic acid (RA)-dependent pathway. Splenic cDCs isolated from C57BL/6 mice were either left uninfected or infected with Ad5 (non-recombinant) for $8 \mathrm{~h}$. Following infection, cells were washed and co-cultured with OT-I T cells (CD8) in the presence of the ovalbumin (OVA) peptide for $72 \mathrm{~h}$. (a) Representative FACS plots (left panel) showing cell surface expression of $\alpha_{4} \beta_{7}$ on OT-I T cells 3 days after co-culture. Cells were gated on total CD8 T cells. (Middle panel) Mean frequency of $\alpha_{4} \beta_{7}{ }^{+}$cells (gated on total live CD8 lymphocytes) following co-culture with either uninfected cDCs or Ad5-infected cDCs at indicated multiplicities of infection (MOIs). (Right panel) Total live (Via-Probe negative) CD8 T-cell number at the end of the co-culture primed by cDCs infected with different MOIs. (b) Mean frequency of CCR9 ${ }^{+}$cells (gated on total live CD8 lymphocytes) following co-culture with either uninfected cDCs or Ad5-infected cDCs at MOI 2. (c) Representative fluorescence-activated cell sorting (FACS) plots (left panel) showing $\alpha_{4} \beta_{7}$ expression on total live CD8 T cells following co-culture with Ad5-infected cDCs (MOI 0.15) in the absence or presence of retinoic acid receptor (RAR) inhibitor LE540. (Middle panel) Frequency of $\alpha_{4} \beta_{7}{ }^{+}$CD8 T cells in the absence or presence of LE540 (inhibitor included in the co-culture). Total live (Via-Probe negative) CD8 T-cell number in the absence or presence of LE540 at the end of the co-culture. Data from two independent experiments (Exp. 1 and Exp. 2) are shown. (Right panel) Frequency of $\alpha_{4} \beta_{7}{ }^{+}$CD8 T cells with RAR inhibitor included either only during $8 \mathrm{~h}$ of Ad5 infection and washed before co-culture (pre-treat) or left in the well during co-culture for $72 \mathrm{~h}$ (in well). (d) Quantitative real-time PCR (RT-PCR) showing the fold change (mean of six independent experiments) in the expression of mRNA encoding retinal dehydrogenase (RALDH) enzymes (Aldh1a1, Aldh1a2, and Aldh1a3) in Ad5-infected cDCs over uninfected cDCs at 0, 6-12, and 24h following in vitro infection.

(e) Representative FACS plots (left panel) showing aldehyde dehydrogenase (ALDH)-positive cells (gated on total CD11C + cells) at $8 \mathrm{~h}$ following in vitro Ad5 infection. The ALDH + gate was determined based on the DEAB (4-diethylamino benzaldehyde)-treated uninfected sample. (Right panel) Cumulative data (mean) for three independent experiments. (f) Mean frequency of $\alpha_{4} \beta_{7}{ }^{+}$CD8 T cells following co-culture with either uninfected cDCs or Ad5-infected cDCs in the absence or presence of a RALDH inhibitor, DEAB (left panel). (Right panel) Total live (Via-Probe negative) CD8 T-cell number in the absence or presence of DEAB at the end of the co-culture. (g) C57BL/6 mice were immunized with Ad5 (5×107 PFU per mouse) intramuscularly and draining lymph nodes (DLNs; inguinal and popliteal pooled) were harvested at $12 \mathrm{~h}$ postimmunization. Quantitative real-time PCR (RT-PCR) showing the fold change in the expression of mRNA encoding RALDH enzymes (Aldh1a1, Aldh1a2, and Aldh1a3) in cDCs from Ad5-infected mice DLNs over naive mice DLNs. Results from two independent experiments are shown (Exp. 1 and Exp. 2). Error bars indicate s.e.m. All data (except Figure 2g) are representative of at least three independent experiments. ${ }^{\star} P<0.05$; ${ }^{\star \star} P<0.01$; ${ }^{\star \star \star} P<0.001$. The $P$-values indicate significantly higher responses compared with the respective uninfected DC controls.

promoter regions of target genes to regulate transcription. ${ }^{12,13}$ Inclusion of the RAR inhibitor LE540 in the co-cultures of Ad5-infected peripheral cDCs and OT-I T cells abrogated the $\alpha_{4} \beta_{7}$ expression on OT-I T cells (Figure 2c), suggesting that Ad5-mediated induction of $\alpha_{4} \beta_{7}$ on antigen-specific CD8 T cells is RA dependent. The viable OT-I T-cell number at the end of the co-culture was similar in the absence and presence of inhibitor, indicating that loss of $\alpha_{4} \beta_{7}$ expression on OT-I cells in the presence of inhibitor was not due to cell death. Furthermore, inclusion of LE540 during the Ad5 infection period, but not in co-culture, did not diminish the percentage of $\alpha_{4} \beta_{7}$-positive OT-I T cells, suggesting that inhibition of RAR in Ad5-infected 
cDCs may not significantly affect their ability to induce guthoming potential on OT-I T cells (Figure 2c, right panel). Thus, our working hypothesis is that upon Ad5 infection, peripheral cDCs acquire the ability to synthesize RA and the RA acts on T cells to induce $\alpha_{4} \beta_{7}$ upregulation.

Previous studies have indicated that it is difficult to measure retinol metabolites in the supernatants of DC cultures as they are made in very small quantities. ${ }^{14}$ Hence, we resorted to measure the levels of RALDH, a key enzyme that is required for the synthesis of RA. There are three isozymes for RALDH that are encoded by Aldh1a1, Aldh1a2, and Aldh1a3 genes. We quantitated the effect of Ad5 infection on the induction of mRNA for these three genes in splenic cDCs and found a 1.3to 1.7-fold increase of Aldh1a1, Aldh1a2, and Aldh1a3 message at 6-12 $\mathrm{h}$ postinfection in Ad5-infected over Ad5-uninfected cDCs (Figure 2d). We further validated the activity of aldehyde dehydrogenase (ALDH) protein in individual cells using a fluorescent substrate (ALDEFLUOR) for ALDH. Following in vitro infection with Ad5, the percentage of ALDH + cells in Ad5-infected cDCs was 1.7- and 2.3-fold higher in comparison with the uninfected cells at 8 and $16 \mathrm{~h}$, respectively (Figure 2e). Also, in line with our hypothesis, inclusion of an ALDH inhibitor DEAB (4-diethylamino benzaldehyde) in co-cultures abrogated the ability of Ad5-infected cDCs to induce gut-homing potential on OT-I T cells (Figure 2f). Viable OT-I T-cell numbers at the end of culture confirmed that the DEAB-mediated abolishment of $\alpha_{4} \beta_{7}$ expression is not an artifact because of excessive cell death. To confirm that Ad5 induces expression of RALDH genes in vivo, we sorted cDCs from the DLNs of mice following IM immunization with Ad5. Consistent with in vitro data in splenic cDCs, we observed an upregulation of Aldh1a2 by 1.3- to 2.9 -fold and Aldh1a3 by 1.5-fold in Ad5-immunized DLN cDCs over naive control mice DLN cDCs at $12 \mathrm{~h}$ postimmunization (Figure 2g).

\section{Inactivated Ad5 also endows splenic CDCs with the capacity to imprint gut-homing potential on antigen-specific CD8T cells}

To investigate whether live Ad5 is required for the ability of $\mathrm{cDCs}$ to induce gut-homing potential on antigen-specific CD8 T cells, we infected splenic DCs with either ultraviolet (UV)-inactivated or heat-inactivated Ad5. UV inactivation is known to inhibit viral transcription and heat inactivation is known to inhibit viral entry. ${ }^{15} \mathrm{UV}$ or heat inactivation of the virus resulted in a minimum of 5-log reduction in the viral titer (data not shown). Interestingly, neither UV nor heat inactivation diminished the ability of Ad5 to modulate $\mathrm{CDC}$ function to imprint gut-homing potential on OT-I $\mathrm{T}$ cells (Figure $3 \mathrm{a}$ ). In fact, we observed a marginally higher percentage of $\alpha_{4} \beta_{7}+$ CD8 T cells primed with DCs pulsed with either of the inactivated Ad5 in comparison with those primed with live Ad5-infected DCs. Consistent with the priming of $\alpha_{4} \beta_{7}+$ CD8 $\mathrm{T}$ cells, we also observed induction of ALDH activity in CDCs by 16-24h following stimulation with UV- or heat-inactivated Ad5 (Figure 3b). Notably, in contrast to live Ad5, the inactivated Ad5s induced ALDH + cells with a delayed kinetics.

Murine DCs lack CAR (Coxsackievirus and adenovirus receptor) that is needed for Ad5 infectivity, suggesting that Ad5 does not efficiently infect murine cDCs. To confirm this, we infected splenic cDCs with an Ad5-expressing green fluorescent protein (Ad5-GFP) at a MOI of 2 for $24 \mathrm{~h}$ and found that $<3 \%$ of the total $\mathrm{cDC}$ population was positive for GFP (Figure 3c). The low level of infection could be because of CAR-independent rAd5 tropism that has been reported previously. ${ }^{16-18}$ These results demonstrate that productive Ad5 infection per se may not be important as UVor heat-inactivated Ad5 could also modulate DC function. We speculate that mere Ad5 binding to the DCs is probably engaging multiple pattern recognition receptors and/or cell surface integrins such as $(\alpha \mathrm{v} \beta 1, \alpha v \beta 3, \alpha v \beta 5)^{19}$ and that downstream signaling through these upregulates RALDH genes.

\section{Signaling through multiple pattern recognition receptors is dispensable for Ad5-infected cDCs to imprint gut-homing potential on CD8 $\mathrm{T}$ cells}

Stimulation through TLR2 on DCs by zymosan can induce Aldh $1 a 2,{ }^{20}$ and Ad5 is known to engage multiple pattern recognition receptors, including the TLRs. ${ }^{21}$ Thus, we investigated whether signaling through the TLRs on $\mathrm{cDCs}$ is required for Ad5-mediated enhancement of $\mathrm{cDC}$ function to prime guthoming potential on CD8 T cells. DCs from both $M y D 88^{-/-}$as well as $T R I F^{-1-}$ mice were as efficient as DCs from wild-type $\mathrm{B} / 6$ mice to induce $\alpha_{4} \beta_{7}$ expression on CD8 T cells following infection with Ad5, demonstrating that TLR signaling is not required for the ability of Ad5 to modulate $\mathrm{CDC}$ function (Figure 4a). In addition, we detected the Ad5-mediated induction of RALDH message by quantitative real-time PCR in cDCs from these knockout mice (data not shown), thus suggesting against any synergism between TLR signaling and RA metabolism in Ad5-infected cDCs. Interestingly, in the absence of Ad5, we noted a marked decrease in proliferation of OT-I T cells in cultures with cDCs from the knockout mice (Supplementary Figure S1 online); however, Ad5 infection restored this defect.

Only TLR4 is known to activate both the TIRAP/MyD88 (TIR-domain containing adapter protein/myeloid differentiation primary response gene (88)) and the TRAM/TRIF (TRIF-related adapter molecule/TIR-domain-containing adapter-inducing interferon- $\beta$ ) signaling pathways, ${ }^{22,23}$ unlike all other known TLRs that use either MyD88 or TRIF as a downstream adaptor molecule. ${ }^{24,25}$ To rule out any role of TLR4 signaling in RA metabolism following Ad5 infection, we used cDCs from $T L R 4^{-/-}$mice. Ad5-infected splenic cDCs from TLR $4^{-/-}$mice were as efficient as $\mathrm{CDC}$ from wild-type $\mathrm{B} / 6$ mice in inducing $\alpha_{4} \beta_{7}$ expression on CD8 $\mathrm{T}$ cells (Figure $4 \mathbf{b}$ ), thus ruling out a role for TLR4.

Ad5 is a non-enveloped double-stranded DNA virus, and viral DNA recognition is known to trigger host innate immune responses. Intracellularly, viral DNA can be recognized either by endosomal TLR9 or by a cytosolic DNA sensor called the DAI (previously known as ZBP1). ${ }^{26}$ To investigate the role of Ad5 DNA recognition by splenic $\mathrm{cDCs}$ for induction of gut-homing potential on CD8 T cells, we used cDCs from $Z b p 1^{-/-}$mice in our in vitro assay. Ad5-infected cDCs from $Z b p 1^{-/-}$mice could prime $\alpha_{4} \beta_{7}+$ CD8 T cells with similar efficiency as those from 
a

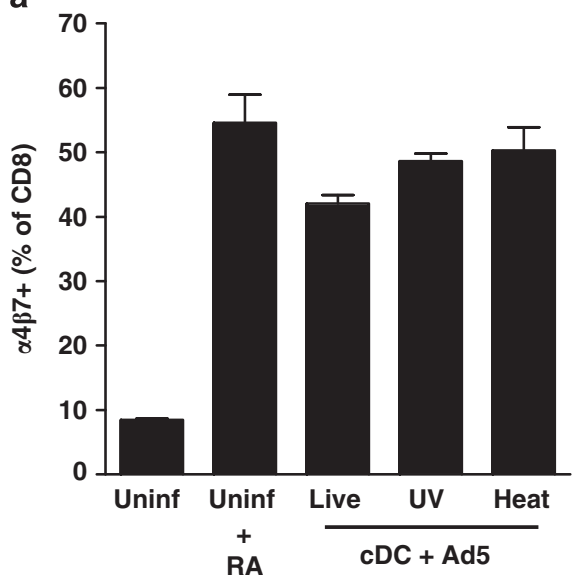

c
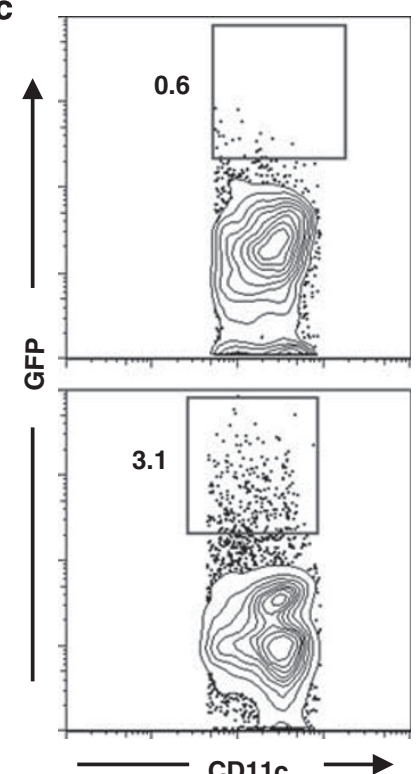

Ad-GFP

inf. $\mathrm{CDC}$

(MOI 2) b
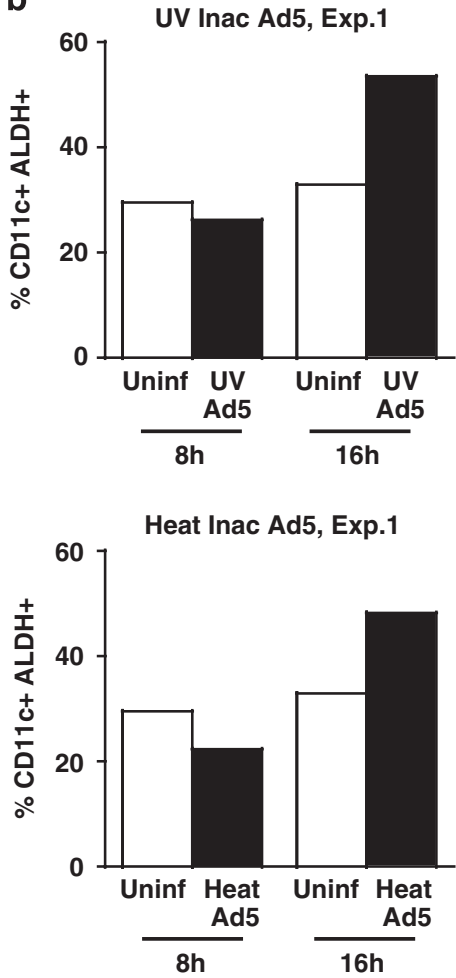

UV Inac Ad5, Exp.2
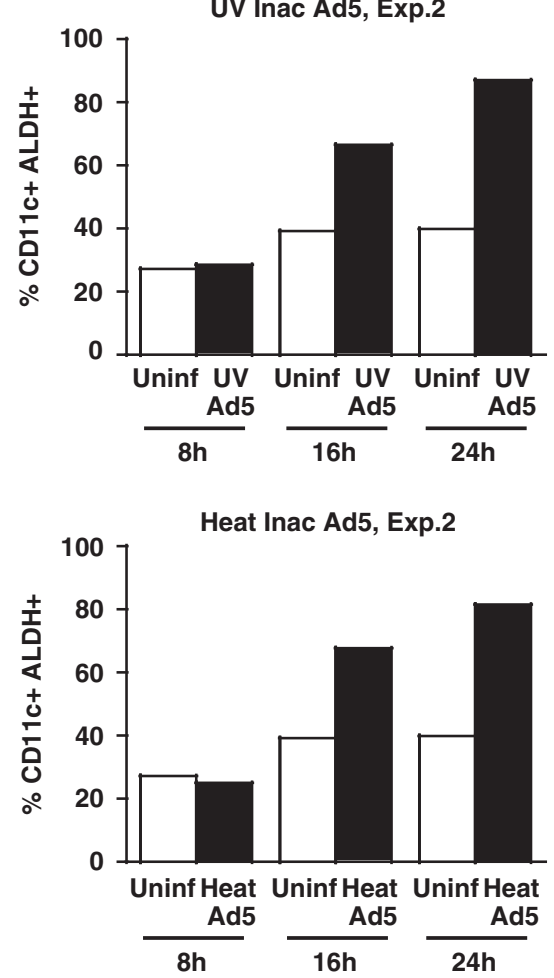

Figure 3 Inactivated adenovirus type 5 (Ad5) also endows splenic conventional dendritic cells (cDCs) with the capacity to imprint gut-homing potential on antigen-specific CD8 T cells. Following isolation of splenic cDCs from wild-type C57BL/6 mice, they were either left uninfected or infected with Ad5 (live) or ultraviolet (UV)/heat-inactivated Ad5 (multiplicity of infection (MOI) 0.15 ) for $8 \mathrm{~h}$. After infection, cells were washed and cultured with OT-I T cells (CD8) in the presence of ovalbumin (OVA) peptide for $72 \mathrm{~h}$. (a) Mean frequency of $\alpha_{4} \beta_{7}{ }^{+}$CD8 T cells following co-culture with either uninfected cDCs or live or inactivated Ad5-infected/stimulated cDCs. (b) Frequency of aldehyde dehydrogenase (ALDH)-positive cells. Data from two independent experiments (Exp. 1 and Exp. 2) are shown. (c) Splenic cDCs from wild-type C57BL/6 mice were infected with Ad5-GFP. After 24h of infection, cells were harvested and analyzed by fluorescence-activated cell sorting (FACS) to detect relative (to uninfected cDCs) levels of expression of green fluorescent protein (GFP; $y$ axis on flow plot). Error bars indicate s.e.m. Data from one of the three independent experiments (except for Figure $\mathbf{3 b}$ ) are shown.

wild-type B/6 mice (Figure 4c). Similar results were also obtained when cDCs from TLR9 $\%$ mice were used (data not shown). These results demonstrate that Ad5-mediated modulation of $\mathrm{cDC}$ function to imprint gut-homing potential on CD8 $\mathrm{T}$ cells is independent of TLR and DAI signaling.

Also, the inclusion of YVAD-cmk ( $N$-Ac-Tyr-Val-Ala-Aspchloromethyl ketone, a caspase-1 inhibitor) during Ad5 infection did not attenuate the ability of cDCs to imprint gut-homing potential on OT-I T cells (Figure 4d). This may rule out any possible involvement of caspase- 1 inflammasomes (particularly AIM2 (absent in melanoma 2), a cytoplasmic inflammasome for double-stranded DNA) or proinflammatory interleukin-1 $\beta .27,28$ However, further studies in ASC (apoptosis-related speck-like protein; an adaptor molecule that links many upstream NODlike receptors) knockout mice can conclusively delineate the role of inflammasomes in Ad5-mediated effect on cDCs with respect to RA metabolism.

DC function can be regulated by multiple MAP kinase pathways such as extracellular-signal-regulated kinase, p38, and c-Jun N-terminal kinase, and Ad5 infection is known to activate these kinases. ${ }^{29}$ To investigate any role of MAP kinase (MAPK) activation in modulating splenic $\mathrm{CDC}$ function, we treated Ad5-infected cDCs with specific MAPK inhibitors UO126 (for MEK1/2), SB203580 (for p38 MAPK), and SP600125 (for c-Jun $\mathrm{N}$-terminal kinase). Interestingly, inclusion of MAPK inhibitors during Ad5 infection did not affect the ability of $\mathrm{cDC}$ to imprint 
a

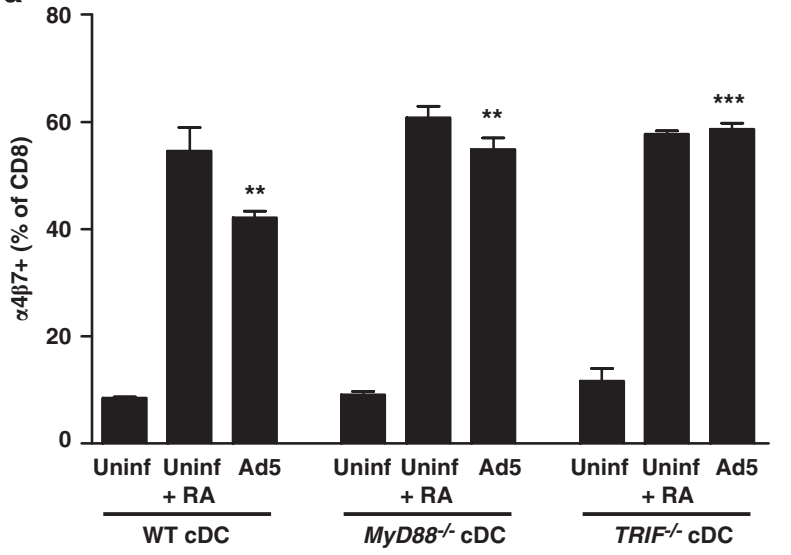

b

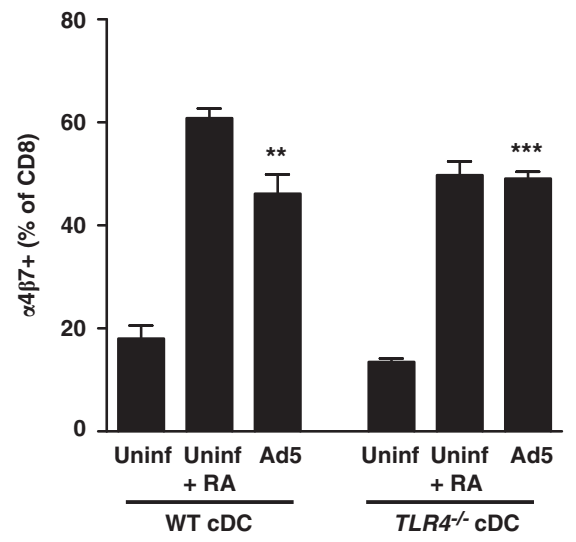

e

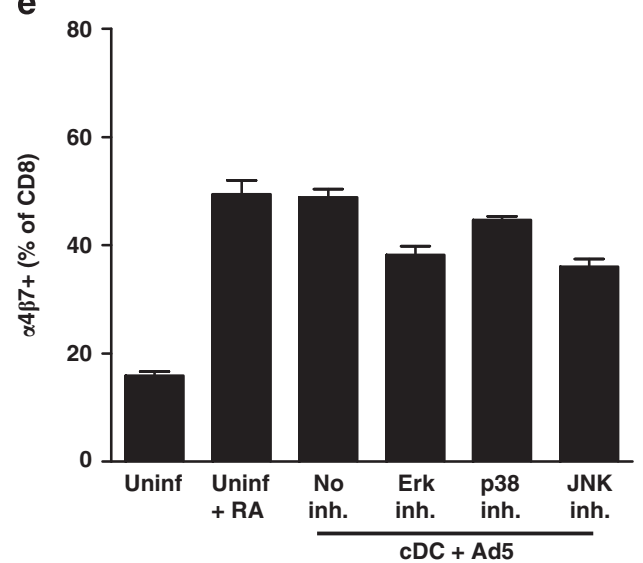

Figure 4 Signaling through multiple pattern recognition receptors (PRRs) is dispensable for adenovirus type 5 (Ad5)-infected conventional dendritic cells (cDCs) to imprint gut-homing potential on CD8 T cells. Splenic cDCs were isolated from either wild-type (WT) C57BL/6 mice or different gene knockout mice and infected with Ad5 (multiplicity of infection (MOI) 0.15) for $8 \mathrm{~h}$, after which infected cells were washed and co-cultured with OT-I T cells (CD8) in the presence of the ovalbumin (OVA) peptide for $72 \mathrm{~h}$. (a) Mean frequency of $\alpha_{4} \beta_{7}{ }^{+}$CD8 T cells following co-culture with either uninfected cDCs or Ad5-infected cDCs isolated from either WT C57BL/6 mice or MyD88 ${ }^{-/-}$or TRIF ${ }^{-/-}$mice. (b) Mean frequency of $\alpha_{4} \beta_{7}^{+}$CD8 T cells following co-culture with either uninfected cDCs or Ad5-infected cDCs isolated from either WT C57BL/6 mice or TLR4 ${ }^{-/-}$mice. (c) Mean frequency of $\alpha_{4} \beta_{7}{ }^{+}$ CD8 T cells following co-culture with either uninfected cDCs or Ad5-infected cDCs isolated from either WT C57BL/6 mice or Zbp $1^{-/-}$mice. (d) Mean frequency of $\alpha_{4} \beta_{7}{ }^{+}$CD8 T cells following co-culture with Ad5-infected cDCs from WT C57BL/6 mice in the presence or absence of caspase- 1 inhibitor YVAD-cmk. (e) Mean frequency of $\alpha_{4} \beta_{7}{ }^{+}$CD8 T cells following co-culture with Ad5-infected cDCs from WT C57BL/6 mice in the presence or absence of extracellular-signal-regulated kinase (ERK) inhibitor (UO126), p38 mitogen-activated protein kinase (MAPK) inhibitor (SB203580), or c-Jun $\mathrm{N}$-terminal kinase (JNK) inhibitor (SP600125). All inhibitors were included only during the $8 \mathrm{~h}$ of Ad5 infection and were excluded from the $72 \mathrm{~h}$ of co-culture. Error bars indicate s.e.m. Data from one of the three (for Figure 4a) or one of two (for Figure $4 \mathbf{b}-\mathbf{e}$ ) independent experiments are shown. ${ }^{\star \star} P<0.01 ;{ }^{* \star \star} P<0.001$. The $P$-values indicate significantly higher responses compared with the respective uninfected DC controls.

gut-homing specificity on OT-I T cells (Figure 4e), thus demonstrating the lack of involvement of these MAPKs.

\section{NF- $\kappa B$ activation in $\mathrm{CDCs}$ is crucial for the priming of gut-homing CD8 T cells following Ad5 infection}

Several studies have shown that DC activation and maturation is closely associated with NF- $\mathrm{KB}$ induction. ${ }^{30,31}$ Ad5 infection (both in mice and humans) is known to trigger rapid production of proinflammatory cytokines such as tumor necrosis factor$\alpha$ that in turn is a potent activator of NF- $\kappa B .{ }^{32,33}$ We next sought to determine if intracellular activation of NF- $\kappa B$ had any role in modulating Ad5-infected $\mathrm{CDC}$ function to prime gut-homing CD8 $\mathrm{T}$ cells. Inclusion of a potent NF- $\kappa \mathrm{B}$ inhibitor NAC ( $N$-acetyl-L-cysteine) ${ }^{34}$ during the Ad5 infection of cDCs (but not in co-culture) significantly reduced the percentage of $\alpha_{4} \beta_{7}$-positive OT-I cells (Figure 5a, left panel), suggesting that Ad5-mediated enhancement of CDC function to imprint gut-homing potential is dependent on activation of the NF- $\mathrm{kB}$. Viable OT-I T cell number at the end of co-culture suggested that the NAC-mediated reduction in $\alpha_{4} \beta_{7}$ expression was not an erroneous observation because of excessive cell death (Figure $\mathbf{5 a}$, right panel). To further confirm the role of NF- $\kappa \mathrm{B}$, we used $\mathrm{cDCs}$ from $\mathrm{N}_{\mathrm{kbl}} \mathrm{1}^{-1-}$ mice in our in vitro assay. Consistent with the results obtained with the NF- $\kappa B$ inhibitor, Ad5 infection failed to enhance priming of $\alpha_{4} \beta_{7}+$ CD8 T cells by cDCs from $N f k b 1^{-1-}$ mice (Figure 5b, left panel). Interestingly, in the absence of Ad5, we noted a marked decrease in proliferation of OT-I T cells in cultures with cDCs from $\mathrm{Nfkb1}^{-/-}$mice (Figure 5b, right panel). However, Ad5 infection restored this defect, suggesting that the failure to upregulate $\alpha_{4} \beta_{7}$ by CD8 T cells in 

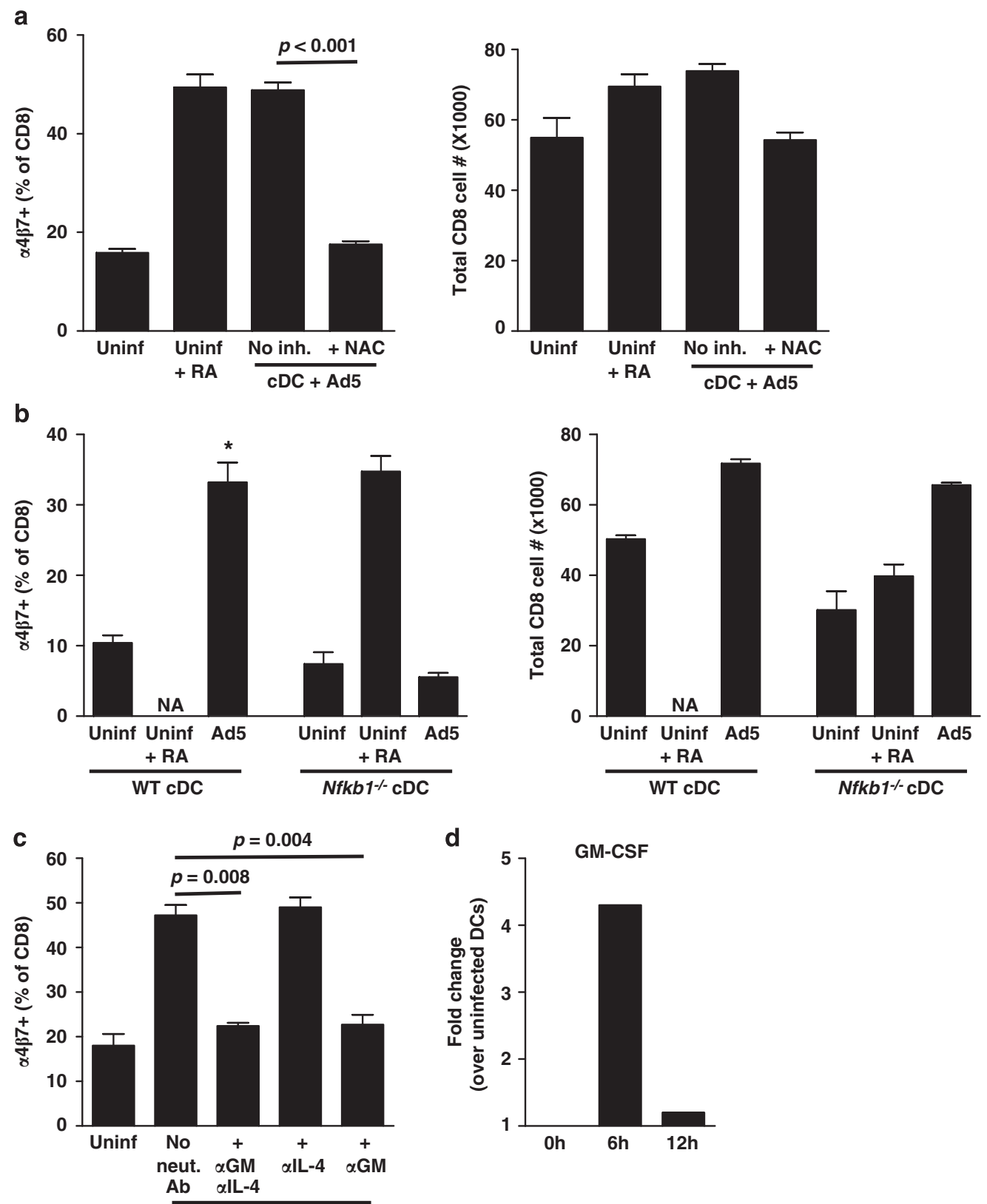

Figure 5 Nuclear factor- $\mathrm{KB}(\mathrm{NF}-\kappa \mathrm{B})$ activation in conventional dendritic cells (cDCs) is crucial for the priming of gut-homing CD8 T cells following adenovirus type 5 (Ad5) infection. Splenic cDCs from either wild-type (WT) C57BL/6 mice or Nfkb1-/- mice were infected with Ad5 (multiplicity of infection (MOI) 0.15) for $8 \mathrm{~h}$, after which infected cells were washed and co-cultured with OT-I T cells (CD8) in the presence of the ovalbumin (OVA) peptide for $72 \mathrm{~h}$. (a) Mean frequency of $\alpha_{4} \beta_{7}{ }^{+}$CD8 T cells following $72 \mathrm{~h}$ of co-culture with Ad5-infected cDCs (from WT C57BL/6 mice) in the presence or absence of NF-kB inhibitor, NAC ( $N$-acetyl-L-cysteine; left panel). (Right panel) Total live (Via-Probe negative) CD8 T-cell number at the end of the co-culture. The inhibitor was included only during the $8 \mathrm{~h}$ of Ad5 infection and was excluded from the co-culture. (b) Mean frequency of $\alpha_{4} \beta_{7}{ }^{+}$cells (gated on total live CD8 lymphocytes) following $72 \mathrm{~h}$ of co-culture is shown on the left and total live (via-probe negative) CD8 T-cell number at the end of the co-culture is shown on the right. (c) Mean frequency of $\alpha_{4} \beta_{7}{ }^{+}$CD8 T cells following co-culture with Ad5-infected cDCs from WT C57BL/6 mice in the presence or absence of neutralizing antibody $(\mathrm{Ab})$ to granulocyte-macrophage colony-stimulating factor (GM-CSF; MP1-22E9) or interleukin-4 (IL-4; 11B11) or both. Neutralizing Abs were included in the $72 \mathrm{~h}$ of co-culture. (d) Quantitative real-time PCR (RT-PCR) showing the fold change in the expression of mRNA encoding GM-CSF (Csf2) in Ad5-infected cDCs over uninfected cDCs at 0,6 , and $12 \mathrm{~h}$ following in vitro infection. Error bars indicate s.e.m. Data from one of the two (for Figure 5a-c) or one of three (for Figure $5 \mathrm{~d}$ ) independent experiments are shown. ${ }^{\star} P<0.05$. The $P$-values indicate significantly higher responses compared with the respective uninfected DC controls. NA, not available.

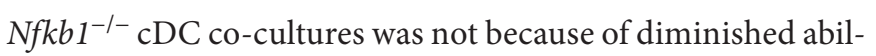
ity of these DCs to get activated and present antigen.

GM-CSF, in synergism with interleukin-4, has been shown to induce RA-producing capacity in bone marrow-derived and splenic cDCs in mice. ${ }^{35} \mathrm{NF}-\mathrm{\kappa B}$ can also regulate GM-CSF expression. ${ }^{36}$ We thus further investigated the role of GMCSF in Ad5-mediated upregulation of $\alpha_{4} \beta_{7}$ on CD8 T cells. Interestingly in co-cultures with Ad5-infected cDCs and OT-I 
T cells, neutralization of GM-CSF resulted in reduction of $\alpha_{4} \beta_{7}$ expression on OT-I T cells to a level that was comparable with uninfected cDCs (Figure 5c). Neutralization of interleukin-4 did not diminish Ad5-mediated effect, either alone or in combination with GM-CSF. In addition, the levels of GM-CSF message increased by 4.3 -fold in the infected splenic $\mathrm{CDCs}$ over uninfected controls at $6 \mathrm{~h}$ following infection (Figure 5d). Our data thus suggest that Ad5-mediated effect can be either directly through upregulation of RALDH enzymes in cDCs or indirectly through GM-CSF or maybe both synergistically involving NF- $\mathrm{\kappa B}$.

\section{DISCUSSION}

Tissue-specific lymphocyte trafficking is regulated by the expression of different homing receptors on these cells ${ }^{11}$ and the tissue-resident DCs have been shown to imprint this property on them. ${ }^{37}$ The gut-resident DCs possess the property of metabolizing vitamin $\mathrm{A}$ to $\mathrm{RA}$ that in turn regulates expression of the guthoming receptor $\alpha_{4} \beta_{7}$ on T and B cells. ${ }^{3}$ Our results demonstrate that the live replication-deficient vector $\mathrm{Ad} 5$ can induce expression of vitamin A-metabolizing enzymes in peripheral DLN DCs in vivo and also in splenic DCs in vitro, and that correlates with the ability of this virus to prime gut-homing CD8 T cells. These results provide an innate mechanism by which Ad5 modulates DLN and splenic DC function to prime gut-homing T cells. To our knowledge, this is the first study that shows induction of vitamin A-metabolizing enzymes in non-gut-associated DCs by a replication-deficient viral vector. These results have important implications for developing mucosal vaccines.

Our results demonstrate that NF- $\kappa \mathrm{B}$ activation in $\mathrm{cDCs}$ is essential for Ad5-mediated induction of their ability to prime gut-homing CD8 T cells. Ad5 is a potent inducer of NF- $\mathrm{KB}^{38}$ and studies have demonstrated the presence of NF- $\mathrm{\kappa B}$-binding sites in both the murine Aldh $1 a 2^{39}$ and the GM-CSF (Csf2 $)^{36}$ promoter region, thus suggesting that NF- $\mathrm{kB}$ is a transcription activator for both genes. Our data with neutralizing antibody to GM-CSF also showed that this cytokine can modulate the ability of Ad5-infected splenic cDC to induce expression of $\alpha_{4} \beta_{7}$ on CD8 T cells. As reported earlier, ${ }^{35} \mathrm{GM}-\mathrm{CSF}$ made by lamina propria macrophages or granulocytes is known to positively regulate Aldh1a2 expression in gut DCs. The same study also showed that RA in-turn can upregulate the Csf2 expression in mesenteric lymph node-resident non-DCs. As evident from the quantitative real-time PCR data in our study, Ad5 infection can induce both RALDH enzymes and Csf 2 in the splenic cDCs by as early as $6-12 \mathrm{~h}$ following infection. Thus, in the context of Ad5, as is true for the gut microenvironment, we can speculate that RA and GM-CSF have a synergistic effect on modulating CDC function with respect to priming of gut-homing CD8 T cells. Ad5 immunization studies in GM-CSF knockout mice (Beta$\mathrm{c}^{--}$) should further validate the role of GM-CSF in Ad5-mediated induction of gut homing of CD8 $\mathrm{T}$ cells.

Although Ad5 is known to induce NF- $\kappa B,{ }^{38}$ yet, the mechanism/s leading to NF- $\kappa B$ activation are yet to be defined. $\mathrm{NF}-\kappa \mathrm{B}$ can be induced by many pattern recognition receptors including TLRs, retinoic acid-inducible gene I (RIG-I), and DAI. Of these, being a double-stranded DNA enveloped virus, it is likely that Ad5 could target TLRs and DAI. However, our results demonstrated that signaling through TLRs and DAI in splenic cDCs is not necessary for the Ad5-mediated induction of their ability to prime gut-homing $\mathrm{CD} 8 \mathrm{~T}$ cells. It is interesting to note that the cDCs from these knockout mice were poor at stimulating OT-I T cells in comparison with cDCs from wild-type B/6 mice, and Ad5 restored this defect (Supplementary Figure S1 online), suggesting that Ad5 can activate cDCs in a TLR- and DAI-independent manner. A similar situation was also observed with cDCs from $\mathrm{Nfkb1}^{-1-}$ mice, demonstrating that the failure of these DCs to prime $\alpha_{4} \beta_{7}$-positive OT-I T cells was not due to lack of DC activation (Figure 5b).

Vitamin A can be metabolized by any one of the three isoforms of the RALDH enzyme. Our results showed that Ad5 induces expression of primarily Aldh $1 a 2$ and Aldh $1 a 3$ genes, and it is not clear whether both these isoforms are needed for the Ad5mediated induction of gut-homing CD8 T cells by splenic DCs. The TLR2 ligand, zymosan, is also known to induce Aldh1a2; ${ }^{20}$ however, Ad5-mediated induction of RALDH enzymes was not dependent on TLR signaling. These results suggest that Ad5 is working through a pathway that is different from previously reported TLR-dependent induction of RALDH enzymes in splenic cDCs.

As both DCs and T cells are known to have RARs, we investigated whether the endogenously made RA by the Ad5-infected peripheral cDCs is working in an autocrine manner (thus further conditioning these DCs somehow to prime CD8 T cells to upregulate $\alpha_{4} \beta_{7}$ ) or in a paracrine manner on the CD8 T cells. For example, RA made by zymosan-stimulated DCs has been shown to act on the same DCs in an autocrine manner. ${ }^{20}$ However, in our study, the inclusion of RAR inhibitor only during the $8 \mathrm{~h}$ of Ad5 infection period did not abrogate the $\alpha_{4} \beta_{7}$ expression on CD8 T cells, suggesting that RA made by the Ad5stimulated DCs is working mostly in a paracrine manner.

Our results demonstrate that the cytosolic DNA sensor DAI is not required for Ad5-mediated priming of gut-homing CD8 T cells by splenic DCs, suggesting that Ad5 DNA is not required for this function. It is important to note here that the role of DAI as a cytosolic sensor for viral DNA can be redundant, as other studies in murine model have shown that DAI-deficient mice exhibit uncompromised immune responses following infection with DNA viruses. ${ }^{40,41}$ A recent study using a human cell line has however identified the extra-chromosomal histone $\mathrm{H} 2 \mathrm{~B}$ as biologically crucial for innate immune response to DNA viruses. ${ }^{42}$ Whether histone $\mathrm{H} 2 \mathrm{~B}$ has any role as a cytosolic DNA sensor in the murine model or not needs to be further investigated.

At present, it is unclear which part of Ad5 (either protein or nucleic acid) is responsible for its ability to induce vitamin A-metabolizing enzymes in cDCs. We speculate that this could be one of the Ad5 proteins, as other studies have shown that even empty capsids derived from Ad5 can induce strong inflammatory responses. ${ }^{43,44}$ Identification of this key adenoviral protein/s that can endow peripheral $\mathrm{CDCs}$ with the ability to imprint guthoming potential on antigen-specific CD8 T cells can help in the development of novel mucosal adjuvants for subunit vaccines administered via the parenteral route of immunization. 


\section{METHODS}

Mice and Immunization. For immunization studies, female BALB/c mice of 6-8 weeks of age were purchased from Charles River Laboratories (Willmington, MA). Mice were immunized with $1 \times 10^{6} \mathrm{PFU}$ of recombinant Ad5 expressing HIV-1 clade B Env and Gag (Ad5/Env-Gag) or non-recombinant Ad5 in sterile phosphate-buffered saline in a final volume of $100 \mu \mathrm{l}$ with $50 \mu \mathrm{l}$ injected IM in each of the hind legs at week 0 (prime) and week 4 (boost). For in vitro co-culture assays, C57BL/6, OT-I Tg, and $\mathrm{Nfkb1}^{-1-}$ mice were purchased from Jackson Laboratories (Bar Harbor, Maine). The $\mathrm{Zbp1}^{-{ }^{--}}$mice were pro-

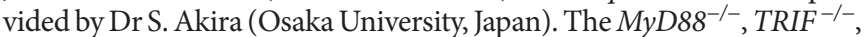
and $T L R 4^{-/-}$mice were bred onsite. All knockout mice were on a $\mathrm{C} 57 \mathrm{BL} / 6$ genetic background. Mice were housed in specific pathogen-free conditions in the Emory Vaccine Center vivarium and were cared for under guidelines established by the Animal Welfare Act and the NIH (National Institutes of Health) "Guide for the Care and Use of Laboratory Animals" using protocols approved by the Emory University institutional animal care and use committee.

Isolation of LPLs. LPLs from small intestines were isolated as described previously. ${ }^{45}$ Briefly, small intestines were removed and Peyer's patches were excised. The intestine was opened up longitudinally and cleaned of all fecal contents. Intestines were cut into small pieces and were transferred into $50 \mathrm{ml}$ conical tubes and shaken at 250 r.p.m. for $20 \mathrm{~min}$ at $37^{\circ} \mathrm{C}$ in Hank's balanced salt solution media (Life Technologies, Carlsbad, CA) supplemented with 5\% fetal bovine serum (Cellgro, Manassas, VA) and 2 mm EDTA (Promega, Madison, WI). After a total of two rounds of EDTA treatment, cell suspensions were passed through a strainer and the remaining intestinal tissue was washed and minced, transferred into $50 \mathrm{ml}$ conical tubes, and shaken for $20 \mathrm{~min}$ at $37^{\circ} \mathrm{C}$ in Hank's balanced salt solution media supplemented with $5 \%$ fetal bovine serum and type VIII collagenase $\left(1.5 \mathrm{mg} \mathrm{ml}^{-1}\right.$; Sigma, St Louis, MO). Cell suspensions were collected and passed through a strainer and were pelleted by centrifugation at 1,500 r.p.m. Mononuclear cells were then isolated using a discontinuous density gradient procedure (45 and 70\%) with Percoll (GE Heathcare Bio-Sciences, Uppsala, Sweden).

Tetramer and intracellular cytokine staining. Tetramer and intracellular cytokine staining was performed as described before. ${ }^{46}$ For tetramer analysis, cells were stained using allophycocyanin-conjugated major histocompatibility complex class I $\left(\mathrm{H}-2 \mathrm{~K}^{\mathrm{d}}\right.$ ) peptide (AMQMLKETI) tetramer (NIH tetramer core facility), CD4-FITC (clone L3T4), CD19-FITC (clone 1D3), $\alpha_{4} \beta_{7}$-phycoerythrin (clone DATK32), CCR9 (clone 242503, R\&D Systems, Minneapolis, MN) and CD8-PerCP (clone 53-6.7). CD8 + CD4- CD19 - and tetramer + cells were scored as tetramer-positive cells. All antibodies were purchased from BD Pharmingen (San Diego, CA).

Analysis of ALDH activity. ALDH activity at a single-cell level was quantified using purified CD11c + cells and ALDEFLUOR staining kit (StemCell Technologies, Vancouver, Canada), according to the manufacturer's protocol with modifications. Briefly, cells $\left(1 \times 10^{6}\right.$ per $\left.\mathrm{ml}\right)$ were suspended in ALDEFLUOR assay buffer containing the activated ALDEFLUOR substrate, with or without the ALDH inhibitor DEAB, and incubated for $45 \mathrm{~min}$ at $37^{\circ} \mathrm{C}$. ALDEFLUOR-reacted cells were scored in fluorescein isothiocyanate (FITC) channel of BD FACSCalibur. Cell viability was determined using the Via-Probe dye (BD Pharmingen).

DC:OT-I co-culture. CD11c ${ }^{+}$DCs from collagenase type IV $\left(1 \mathrm{mg} \mathrm{ml}^{-1}\right.$; Worthington Biochemical, Lakewood, NJ)-digested spleens were isolated using anti-CD11c-coated magnetic beads (N418 clone, Miltenyi Biotec, Bergisch, Germany). The resulting purity of cDCs was $\sim 95 \%$. OT-I T lymphocytes were purified from OT-I spleens using CD8 microbeads (Ly-2 clone, Miltenyi Biotec). The purity of CD8 T cells was $>95 \%$. cDCs and OT-I T cells were co-cultured in a ratio of $1: 2$ for $72 \mathrm{~h}$. To the co-culture, the OVA $_{257-264}$ SIINFEKL peptide was added (20 pM). Where indicated, RA (10 nM; Fluka, St Louis, MO), LE540 (1 $\mu$; Wako, Osaka, Japan),

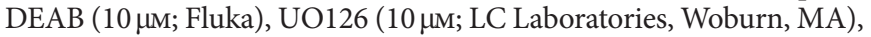

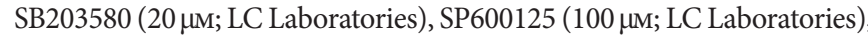
$\mathrm{N}$-acetyl-L-cysteine (30 mM; Sigma), Ac-YVAD-CMK (50 $\mu$; Bachem, King of Prussia, PA), and neutralizing antibody to murine interleukin-4 (clone 11B11; $10 \mu \mathrm{g} \mathrm{ml}^{-1}$, eBioscience), and GM-CSF (clone MP1-22E9; $10 \mu \mathrm{g} \mathrm{ml}^{-1}$, eBioscience, San Diego, CA) was included during either DC infection period or the co-culture. At the end of co-culture, cell surface expression of $\alpha_{4} \beta_{7}$ or CCR9 on total CD8 T cells were analyzed by flow cytometry. The Via-Probe dye (BD Pharmingen) was included in the staining panel to eliminate dead cells.

Adenoviral vectors. The E1/E3-deleted adenovirus type 5 without a transgene (Ad5), or encoding either the HIV-1 clade B Env and Gag (Ad5/Env-Gag) or a GFP (Ad5-GFP) were used. The viral vectors were generated by standard methods as described previously. ${ }^{47}$ Heat inactivation of the virus was achieved by incubation at $56^{\circ} \mathrm{C}$ for $1 \mathrm{~h}$. UV inactivation was achieved by exposing the virus to a $365-\mathrm{nm}$ long UV lamp for $1 \mathrm{~h}$.

Gene expression analysis. The relative quantitative real-time PCR was performed for mRNA expression analyses as described previously. ${ }^{48}$ Total four individual gene expression assays were used from Applied Biosystems (Foster City, CA) for Mus. musculus: aldehyde dehydrogenase family 1, subfamily A1 (Aldh1a1); aldehyde dehydrogenase family 1 , subfamily A2 (Aldh1a2); aldehyde dehydrogenase family 1, subfamily A3 (Aldh1a3); mouse GAPD (GAPDH) Endogenous Control (VIC/MGB Probe). Samples were tested in duplicate. Fold changes were calculated using the comparative $\mathrm{C}_{\mathrm{T}}$ method ${ }^{49}$ using the equation $2^{-\Delta \Delta \mathrm{CT}}$ where $\Delta \Delta \mathrm{C}_{\mathrm{T}}=\left(\left(\mathrm{C}_{\mathrm{T}}\right.\right.$ gene of interest $-\mathrm{C}_{\mathrm{T}}$ gene of control $)$ sample $\mathrm{A}-\left(\mathrm{C}_{\mathrm{T}}\right.$ gene of interest- $\mathrm{C}_{\mathrm{T}}$ gene of control) sample $\left.\mathrm{B}\right)$.

\section{Statistics}

Student's $t$-test was used to compare the differences between groups. The Bonferroni method was used to adjust the $P$-values for multiple comparisons. Statistical analyses were performed using TIBCO Spotfire $S+8.1$ (Palo Alto, CA). A two-sided $P<0.05$ was considered statistically significant.

SUPPLEMENTARY MATERIAL is linked to the online version of the paper at http://www.nature.com/mi

\section{ACKNOWLEDGMENTS}

We are grateful to $\mathrm{Dr}$ Shizuo Akira for kindly providing us with $\mathrm{Zbp1^{-/ }}$ mice. We thank Dr Velu Vijayakumar for help with the flow cytometry. We thank the veterinary staff at the Yerkes Division of Research Resources for their support and H. Drake-Perrow for administrative support. We also thank the Emory CFAR virology core for qPCR measurements and CFAR immunology core for flow cytometry. This work was supported by the National Institutes of Health/National Institute of Allergy and Infectious Diseases grants R01 Al057029 and R01 Al071852 to R.R.A.; U54 Al057157, R37 Al48638, R01 DK057665 to B.P; Yerkes National Primate Research Center base grant, P51 RR00165; Emory CFAR grant P30 Al050409.

\section{DISCLOSURE}

The authors declared no conflict of interest.

() 2011 Society for Mucosal Immunology

\section{REFERENCES}

1. Neutra, M.R. \& Kozlowski, P.A. Mucosal vaccines: the promise and the challenge. Nat. Rev. Immunol. 6, 148-158 (2006).

2. Belyakov, I.M. \& Berzofsky, J.A. Immunobiology of mucosal HIV infection and the basis for development of a new generation of mucosal AIDS vaccines. Immunity 20, 247-253 (2004).

3. Iwata, M. et al. Retinoic acid imprints gut-homing specificity on T cells. Immunity 21, 527-538 (2004).

4. Mora, J.R. et al. Generation of gut-homing IgA-secreting B cells by intestinal dendritic cells. Science 314, 1157-1160 (2006).

5. Guilliams, M. et al. Skin-draining lymph nodes contain dermis-derived CD103(-) dendritic cells that constitutively produce retinoic acid and induce Foxp3(+) regulatory T cells. Blood 115, 1958-1968 (2010). 
6. Lin, S.W., Cun, A.S., Harris-McCoy, K. \& Ertl, H.C. Intramuscular rather than oral administration of replication-defective adenoviral vaccine vector induces specific CD8+ T cell responses in the gut. Vaccine $25,2187-$ 2193 (2007).

7. Tatsis, N. et al. Multiple immunizations with adenovirus and MVA vectors improve CD8+ T cell functionality and mucosal homing. Virology 367 , 156-167 (2007).

8. Kaufman, D.R. et al. Trafficking of antigen-specific CD8+ T lymphocytes to mucosal surfaces following intramuscular vaccination. J. Immunol. 181, 4188-4198 (2008).

9. Masopust, D. et al. Dynamic T cell migration program provides resident memory within intestinal epithelium. J. Exp. Med. 207, 553-564 (2010).

10. Buchbinder, S.P. et al. Efficacy assessment of a cell-mediated immunity HIV-1 vaccine (the Step study): a double-blind, randomised, placebocontrolled, test-of-concept trial. Lancet 372, 1881-1893 (2008).

11. Butcher, E.C. \& Picker, L.J. Lymphocyte homing and homeostasis. Science 272, 60-66 (1996).

12. Balmer, J.E. \& Blomhoff, R. Gene expression regulation by retinoic acid. J. Lipid Res. 43, 1773-1808 (2002).

13. Bastien, J. \& Rochette-Egly, C. Nuclear retinoid receptors and the transcription of retinoid-target genes. Gene 328, 1-16 (2004).

14. Svensson, M. et al. Retinoic acid receptor signaling levels and antigen dose regulate gut homing receptor expression on CD8+ T cells. Mucosal Immunol. 1, 38-48 (2008).

15. Russell, W.C., Valentine, R.C. \& Pereira, H.G. The effect of heat on the anatomy of the adenovirus. J. Gen. Virol. 1, 509-522 (1967).

16. Cheng, C. et al. Mechanism of ad5 vaccine immunity and toxicity: fiber shaft targeting of dendritic cells. PLoS Pathog 3, e25 (2007).

17. Wickham, T.J., Mathias, P., Cheresh, D.A. \& Nemerow, G.R. Integrins alpha $v$ beta 3 and alpha $v$ beta 5 promote adenovirus internalization but not virus attachment. Cell 73, 309-319 (1993).

18. Nemerow, G.R. Cell receptors involved in adenovirus entry. Virology $\mathbf{2 7 4}$, $1-4(2000)$.

19. Jooss, K. \& Chirmule, N. Immunity to adenovirus and adeno-associated viral vectors: implications for gene therapy. Gene Ther 10, 955-963 (2003).

20. Manicassamy, S. et al. Toll-like receptor 2-dependent induction of vitamin A-metabolizing enzymes in dendritic cells promotes T regulatory responses and inhibits autoimmunity. Nat. Med. 15, 401-409 (2009).

21. Hartman, Z.C. et al. Adenovirus infection triggers a rapid, MyD88regulated transcriptome response critical to acute-phase and adaptive immune responses in vivo. J. Virol. 81, 1796-1812 (2007).

22. Yamamoto, M. et al. TRAM is specifically involved in the Toll-like receptor 4-mediated MyD88-independent signaling pathway. Nat. Immunol. 4, 1144-1150 (2003).

23. Kagan, J.C. et al. TRAM couples endocytosis of Toll-like receptor 4 to the induction of interferon-beta. Nat. Immunol. 9, 361-368 (2008).

24. Pulendran, B. Modulating vaccine responses with dendritic cells and Tolllike receptors. Immunol. Rev. 199, 227-250 (2004).

25. Yamamoto, M. et al. Role of adaptor TRIF in the MyD88-independent tolllike receptor signaling pathway. Science 301, 640-643 (2003).

26. Takaoka, A. et al. DAl (DLM-1/ZBP1) is a cytosolic DNA sensor and an activator of innate immune response. Nature 448, 501-505 (2007).

27. Hornung, V. et al. AlM2 recognizes cytosolic dsDNA and forms a caspase-1-activating inflammasome with ASC. Nature 458, 514-518 (2009).

28. Fernandes-Alnemri, T., Yu, J.W., Datta, P., Wu, J. \& Alnemri, E.S. AIM2 activates the inflammasome and cell death in response to cytoplasmic DNA. Nature 458, 509-513 (2009).

29. Tamanini, A. et al. Interaction of adenovirus type 5 fiber with the coxsackievirus and adenovirus receptor activates inflammatory response in human respiratory cells. J. Virol. 80, 11241-11254 (2006).
30. Neumann, M. et al. Differential expression of Rel/NF-kappaB and octamer factors is a hallmark of the generation and maturation of dendritic cells. Blood 95, 277-285 (2000).

31. Rescigno, M., Martino, M., Sutherland, C.L., Gold, M.R. \& RicciardiCastagnoli, P. Dendritic cell survival and maturation are regulated by different signaling pathways. J. Exp. Med. 188, 2175-2180 (1998).

32. Ginsberg, H.S. et al. A mouse model for investigating the molecular pathogenesis of adenovirus pneumonia. Proc. Natl. Acad. Sci. USA 88, 1651-1655 (1991).

33. Beg, A.A., Finco, T.S., Nantermet, P.V. \& Baldwin, A.S. Jr. Tumor necrosis factor and interleukin-1 lead to phosphorylation and loss of I kappa B alpha: a mechanism for NF-kappa B activation. Mol. Cell. Biol. 13, 3301-3310 (1993).

34. Schreck, R., Albermann, K. \& Baeuerle, P.A. Nuclear factor kappa B: an oxidative stress-responsive transcription factor of eukaryotic cells (a review). Free Radic. Res. Commun. 17, 221-237 (1992).

35. Yokota, A. et al. GM-CSF and IL-4 synergistically trigger dendritic cells to acquire retinoic acid-producing capacity. Int. Immunol. 21, 361-377 (2009).

36. Schreck, R. \& Baeuerle, P.A. NF-kappa B as inducible transcriptional activator of the granulocyte-macrophage colony-stimulating factor gene. Mol. Cell. Biol. 10, 1281-1286 (1990).

37. Villablanca, E.J., Russo, V. \& Mora, J.R. Dendritic cell migration and lymphocyte homing imprinting. Histol. Histopathol. 23, 897-910 (2008).

38. Borgland, S.L., Bowen, G.P., Wong, N.C., Libermann, T.A. \& Muruve, D.A. Adenovirus vector-induced expression of the $C-X-C$ chemokine IP-10 is mediated through capsid-dependent activation of NF-kappaB. J. Virol. 74, 3941-3947 (2000).

39. Wang, X., Sperkova, Z. \& Napoli, J.L. Analysis of mouse retinal dehydrogenase type 2 promoter and expression. Genomics 74, 245-250 (2001).

40. Ishii, K.J. et al. TANK-binding kinase-1 delineates innate and adaptive immune responses to DNA vaccines. Nature 451, 725-729 (2008).

41. Wang, Z. et al. Regulation of innate immune responses by DAI (DLM-1/ ZBP1) and other DNA-sensing molecules. Proc. Natl. Acad. Sci. USA $105,5477-5482$ (2008).

42. Kobiyama, K. et al. Extrachromosomal histone H2B mediates innate antiviral immune responses induced by intracellular double-stranded DNA. J. Virol. 84, 822-832 (2010).

43. Kafri, T. et al. Cellular immune response to adenoviral vector infected cells does not require de novo viral gene expression: implications for gene therapy. Proc. Natl. Acad. Sci. USA 95, 11377-11382 (1998).

44. Molinier-Frenkel, V. et al. Immune response to recombinant adenovirus in humans: capsid components from viral input are targets for vectorspecific cytotoxic T lymphocytes. J. Virol. 74, 7678-7682 (2000).

45. Denning, T.L., Wang, Y.C., Patel, S.R., Williams, I.R. \& Pulendran, B. Lamina propria macrophages and dendritic cells differentially induce regulatory and interleukin 17 -producing T cell responses. Nat. Immunol. 8, 1086-1094 (2007).

46. Ganguly, S., Liu, J., Pillai, V.B., Mittler, R.S. \& Amara, R.R. Adjuvantive effects of anti-4-1BB agonist Ab and 4-1BBL DNA for a HIV-1 Gag DNA vaccine: different effects on cellular and humoral immunity. Vaccine $\mathbf{2 8}$, 1300-1309 (2010).

47. Kawakami, Y. et al. Substitution of the adenovirus serotype 5 knob with a serotype 3 knob enhances multiple steps in virus replication. Cancer Res. 63, 1262-1269 (2003).

48. Mandl, J.N. et al. Divergent TLR7 and TLR9 signaling and type I interferon production distinguish pathogenic and nonpathogenic AIDS virus infections. Nat. Med. 14, 1077-1087 (2008).

49. Schmittgen, T.D. \& Livak, K.J. Analyzing real-time PCR data by the comparative C(T) method. Nat. Protoc. 3, 1101-1108 (2008). 